\title{
Complex Langevin and boundary terms
}

\author{
Manuel Scherzer, ${ }^{1, *}$ Erhard Seiler, ${ }^{2, \dagger}$ Dénes Sexty, ${ }^{3,4, *}$ and Ion-Olimpiu Stamatescu ${ }^{1, \S}$ \\ ${ }^{1}$ Institut für Theoretische Physik, Universität Heidelberg, Philosophenweg 16, D-69120 Heidelberg, Germany \\ ${ }^{2}$ Max-Planck-Institut für Physik (Werner-Heisenberg-Institut), \\ Föhringer Ring 6, D-80805 München, Germany \\ ${ }^{3}$ Department of Physics, Bergische Universität Wuppertal, Gaussstrasse 20, D-42119 Wuppertal, Germany \\ ${ }^{4}$ IAS/JSC, Forschungszentrum Jülich, D-52425 Jülich, Germany
}

(Received 13 September 2018; published 24 January 2019)

\begin{abstract}
As is well known the complex Langevin (CL) method sometimes fails to converge or converges to the wrong limit. We identified one reason for this long ago: insufficient decay of the probability density either near infinity or near poles of the drift, leading to boundary terms that spoil the formal argument for correctness. To gain a deeper understanding of this phenomenon, we analyze the emergence of such boundary terms thoroughly in a simple model, where analytic results can be compared with numerics. We also show how some simple modification stabilizes the CL process in such a way that it can produce results agreeing with direct integration. Besides explicitly demonstrating the connection between boundary terms and correct convergence our analysis also suggests a correctness criterion which could be applied in realistic lattice simulations.
\end{abstract}

DOI: $10.1103 /$ PhysRevD.99.014512

\section{INTRODUCTION}

It has been known for a long time that the complex Langevin (CL) method for simulating systems with complex action may fail by either not converging or by converging to the wrong limit. These failures were traced either to insufficient decay of the probability distribution in the complexified configuration space [1-3] (at infinity or at poles of the drift force), or to failure of ergodicity [4,5]. Recently Salcedo [6] has formulated interesting criteria for failure that at first sight seem to be unrelated to the ones identified by us. The most interesting ones derive support properties of the equilibrium measure which are shown in these cases to be in conflict with the correct expectation values.

In this paper we will focus on one such example and show explicitly that the problems are due to slow decay, leading to the appearance of boundary terms in an integration by parts, spoiling the formal proof of correctness. We stress that we are here concerned with the behavior at large noncompact dimensions. The effects of nonholomorphicity have been shown, e.g., in random matrix models

\footnotetext{
*scherzer@thphys.uni-heidelberg.de

†ehs@mpp.mpg.de

*sexty@uni-wuppertal.de

${ }^{\S}$ I.O.Stamatescu@thphys.uni-heidelberg.de
}

Published by the American Physical Society under the terms of the Creative Commons Attribution 4.0 International license. Further distribution of this work must maintain attribution to the author(s) and the published article's title, journal citation, and DOI. Funded by SCOAP .
$[7,8]$ to lead to wrong convergence and were specifically addressed in [4] both in simple models and in QCD.

Here we consider a complex density

$$
\rho(x)=\exp (-S(x)),
$$

periodic with period $2 \pi$ and extending to an entire analytic function without zeroes.

The complex Langevin equation (CLE) in the form used here is

$$
d x=K_{x} d t+d w, \quad d y=K_{y} d t,
$$

where $d w$ is the Wiener process normalized as

$$
\left\langle d w^{2}\right\rangle=2 d t
$$

and the drift is given by

$$
K_{x}=-\operatorname{Re} S^{\prime}(x+i y), \quad K_{y}=-\operatorname{Im} S^{\prime}(x+i y) .
$$

The long time asymptotic average of a generic observable $\mathcal{O}$ is denoted by $\langle\mathcal{O}\rangle_{\infty}$; we say that the CL process yields correct results if this agrees with the "correct" expectation value of the same observable defined as

$$
\langle\mathcal{O}\rangle_{c}=\int d x \mathcal{O}(x) \rho(x),
$$

i.e.,

$$
\langle\mathcal{O}\rangle_{\infty}=\langle\mathcal{O}\rangle_{c}
$$


In $[1,2]$ correctness was derived from the consideration of CL expectation values at finite Langevin time; it was shown that correctness is assured if a certain quantity $F_{\mathcal{O}}(t, \tau)$ is independent of an interpolation parameter $\tau \in[0, t]$, i.e.,

$$
\frac{\partial}{\partial \tau} F_{\mathcal{O}}(t, \tau)=0
$$

Here $F_{\mathcal{O}}(t, \tau)$ interpolates between the correct time evolution $F_{\mathcal{O}}(t, t)=\langle\mathcal{O}(t)\rangle_{0}$ (defined in Sec. III and analyzed in Appendix B) and the time evolution of the expectation of $\mathcal{O}$ under the Langevin process $F_{\mathcal{O}}(t, 0)=\langle\mathcal{O}\rangle_{t}$. The key points are that (7) implies

$$
\langle\mathcal{O}\rangle_{t}=\langle\mathcal{O}(t)\rangle_{0} \quad \forall t>0
$$

and hence,

$$
\lim _{t \rightarrow \infty} F_{\mathcal{O}}(t, t)=\langle\mathcal{O}\rangle_{c}
$$

The left-hand side of (7), by using integration by parts, is found to be equal to a boundary term; explicitly

$$
\frac{\partial}{\partial \tau} F_{\mathcal{O}}(t, \tau)=\lim _{Y \rightarrow \infty} B_{\mathcal{O}}(Y ; t, \tau),
$$

where

$$
\begin{aligned}
& B_{\mathcal{O}}(Y ; t, \tau) \equiv \\
& \quad \int\left[K_{y}(x, Y) P(x, Y ; t-\tau) \mathcal{O}(x+i Y ; \tau)\right. \\
& \left.\quad-K_{y}(x,-Y) P(x,-Y ; t-\tau) \mathcal{O}(x-i Y ; \tau)\right] d x,
\end{aligned}
$$

$P(x, y ; t)$ is the time evolved probability density under the Langevin evolution and $\mathcal{O}(t) \equiv \mathcal{O}(z ; t)$ is the $L_{c}$ evolved observable (see Sec. III and Appendix B).

This form of the boundary term makes clear that correctness requires sufficient decay of the product $K_{y} P \mathcal{O}$ for all Langevin times $t$.

\section{THE MODEL}

The model studied here is defined by the complex density

$$
\rho=\frac{1}{Z(\beta)} \exp [-i \beta \cos (x)]
$$

and has been studied already in 2007 by Stamatescu [9] and in 2008 by Berges and Sexty [10]. The correct expectation values of exponentials ("modes") are

$$
\begin{aligned}
\int d x \exp (i k x) \rho(x) & =\frac{I_{k}(-i \beta)}{I_{0}(-i \beta)} \\
& =(-i)^{k} \frac{J_{k}(\beta)}{J_{0}(\beta)} \neq 0 .
\end{aligned}
$$

It was found in $[9,10]$ that the CL process does not reproduce the correct EV's which, however, can be regained by a certain reweighting procedure (with different observables requiring sometimes different reweightings).

The remarkable fact found by Salcedo [6] is that the static probability distribution $P(x, y) \equiv P(x, y ; \infty)$ for this model can be written down explicitly by solving the time independent Fokker-Planck equation (FPE); it is

$$
P(x, y)=\frac{1}{4 \pi \cosh ^{2}(y)} .
$$

It is the only non-Gaussian example known to us for which a solution of the static FPE has been found in analytic form. Three features of this solution are remarkable:

(1) $P$ is independent of $x$,

(2) $P$ is independent of $\beta$,

(3) $P$ decays as $\exp (-2|y|)$ for large $|y|$; this decay is not sufficient to make the integrals of the modes

$$
\exp (i k(x+i y)), \quad|k| \geq 2
$$

absolutely convergent, in other words, already here we are faced with slow decay.

\section{A. Complex Langevin results}

But first let us demonstrate that (15) is indeed the distribution produced by running a CL simulation for a long time. The drift force is

$$
\begin{aligned}
& K_{x}=\operatorname{Re} \frac{\rho^{\prime}}{\rho}=-\beta \cos (x) \sinh (y) \\
& K_{y}=\operatorname{Im} \frac{\rho^{\prime}}{\rho}=\beta \sin (x) \cosh (y),
\end{aligned}
$$

for the Langevin process Eq. (2).

In Fig. 1 we show the histogram of the converged marginal distribution $P_{y}(y ; t)=\int d x P(x, y ; t)$ in log scale for $\beta=1$, overlaid with the distribution (15). The histogram is obtained from one long trajectory (Langevin time $t \approx 125000$ ). The agreement over about 6 orders of magnitude is convincing. The distribution can also be seen to be independent of $x$, cf. also Fig. 8 .

We also show in Fig. 2 the histograms of $P_{y}(y ; t)$ for various shorter times and $\beta=0.1$, illustrating the convergence as $t \rightarrow \infty$.

As noted by Salcedo [6], it is obvious that the distribution $P(15)$ cannot reproduce the correct expectation values 


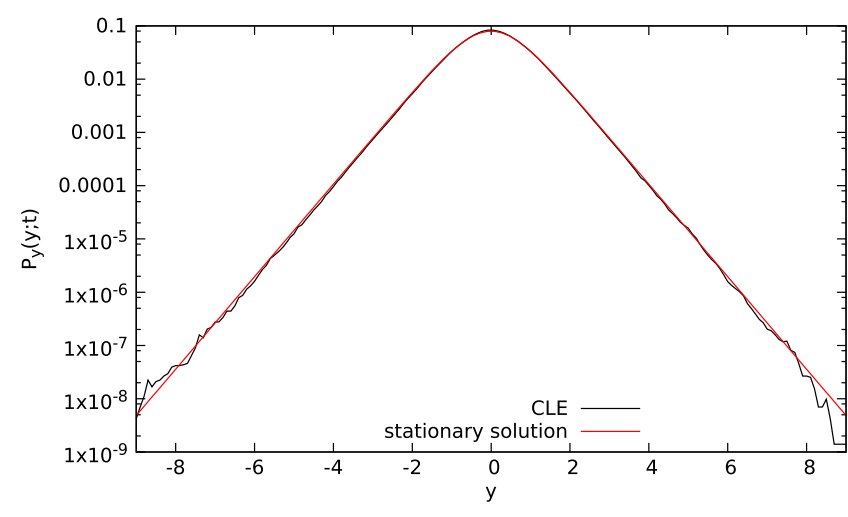

FIG. 1. Comparison of the analytic expression for the marginal distribution $P_{y}(y ; t)$ (15) (red) with the histogram of a CL simulation with $\beta=1$.

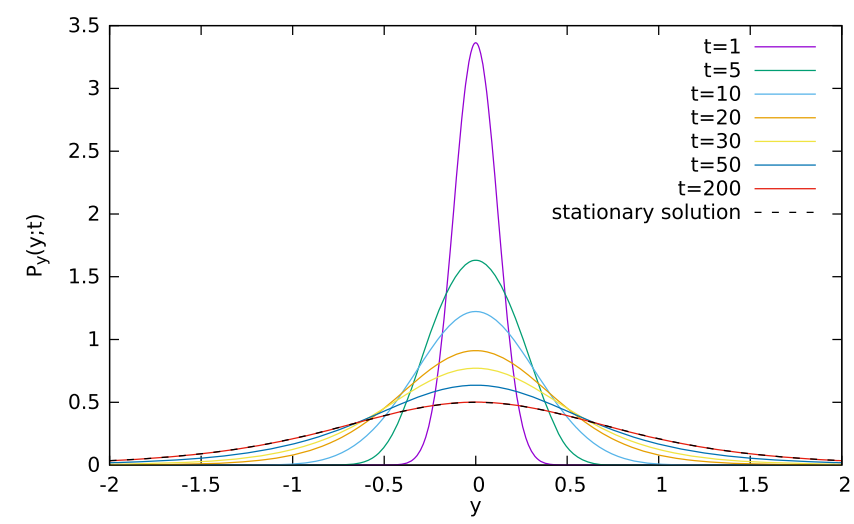

FIG. 2. The marginal distributions $P_{y}(y ; t)$ obtained by numerically solving the FPE for $\beta=0.1$. The ordering of times corresponds to decreasing maxima; note that for $t=200$ no difference is visible between the FPE and the analytical solution.

Eq. (14) of the observables $\mathcal{O}_{k}=\exp (i k x)$, because it is independent of $x$, entailing

$$
\int d x P(x, y) e^{ \pm i k x}=0
$$

and its slow decay makes the expectation values of $\mathcal{O}_{k}$ ill defined for $|k| \geq 2$. In Table I we collect a few CL results, together with the exact expectation values determined by $\rho$ for $\beta=1$. The simulation used 100 independent trajectories

TABLE I. CLE (real part, imaginary part negligible) and correct results for model (13) with $\beta=1$. Last line: "correct evolution" for $t=20$ (see Appendix B).

\begin{tabular}{lccrr}
\hline \hline$\langle\mathcal{O}\rangle$ & $\left\langle\mathrm{e}^{i x}\right\rangle$ & $\left\langle\mathrm{e}^{-i x}\right\rangle$ & \multicolumn{1}{c}{$\left\langle\mathrm{e}^{2 i x}\right\rangle$} & \multicolumn{1}{c}{$\left\langle\mathrm{e}^{-2 i x}\right\rangle$} \\
\hline $\mathrm{CL}$ & $0.004(3)$ & $0.002(3)$ & $1.027(22)$ & $1.001(20)$ \\
Correct & $-0.575081 i$ & $-0.575081 i$ & -0.150162 & -0.150162 \\
$e^{t L_{c}} \mathcal{O}$ & $-0.575081 i$ & $-0.575081 i$ & -0.150162 & -0.150162 \\
\hline \hline
\end{tabular}

with randomly chosen starting points on the real axis, running for a Langevin time of $t \approx 2500$, where measurements were taken after every time step, typically $5 \times 10^{-6}$. The CL values for $|k|>2$ are completely submerged by noise, as expected. For $k= \pm 2$ we find a value close to 1 . It should be remarked that the CL process for $k=2$ evaluates a conditionally convergent integral, so also the measuring schedule plays a role; for instance measuring after every time increment of 0.01 yields very noisy results, consistent with both 0 and 1 . Evaluating the second mode with a fixed cutoff in $y$, we find 0 .

The Schwinger-Dyson equations (SDE)

$$
i k\left\langle e^{i k z}\right\rangle+\frac{\beta}{2}\left\langle e^{i(k+1) z}\right\rangle-\frac{\beta}{2}\left\langle e^{i(k-1) z}\right\rangle=0,
$$

arising from the identity

$$
\int_{-\pi}^{\pi} \rho(x) \mathcal{O}^{\prime}(x) d x=-\int_{-\pi}^{\pi} \rho^{\prime}(x) \mathcal{O}(x) d x
$$

would be satisfied for $k=0, \pm 1$ if the modes \pm 1 are 0 and the modes \pm 2 are 1 , even though these values are not the correct ones.

So the CL results, where they are defined, are incorrect, but mostly_for $|k| \geq 3$, they are completely undefined due to uncontrollable fluctuations.

The last row in the table gives the correct results from the $L_{c}$ evolved observables, as will be explained in the next section. Notice that the correct results of course also satisfy the SDE, but these equations, having the structure of a two-step recursion, have a two-parameter family of solutions $[2,11,12]$.

\section{B. A puzzle}

The remaining question is: how can CL fail for the first mode, i.e., observables $\mathcal{O}_{ \pm 1} \equiv \exp ( \pm i(x+i y))$ ? $\mathcal{O}_{ \pm 1} P$ as well as $K P$ decay exponentially in $y$.

Actually the densities of $\mathcal{O}_{ \pm 1}$ and $K$, if considered not as functions of $y$, but as functions of their actual value decay only powerlike [see Eq. (40)]. Nagata et al. [13] gave an argument that correctness requires exponential decay of the distribution of $K$ and checked their criterion successfully for various cases; so by this criterion correctness is not to be expected here, corroborating the criterion. We will, however, formulate a different criterion in Sec. III, which directly relates to the (non)occurrence of boundary terms.

The CL simulation produces for $\mathcal{O}_{ \pm 1}$ well converged, yet incorrect results, close to 0 [consistent with (15) but inconsistent with (14)].

The resolution lies in the nonvanishing boundary terms arising in the time dependent expectation values and persisting for arbitrarily large times; this is the mechanism described in $[1,2]$. In the following section we will analyze those boundary terms in detail. 


\section{BOUNDARY TERMS FOR FINITE LANGEVIN TIME}

The formal argument for correctness [1,2] is revisited in Appendix A. It requires the choice of an initial distribution $P(x, y ; 0)$; in the following we will choose for simplicity

$$
P(x, y ; 0)=\frac{1}{2 \pi} \delta(y) .
$$

The identity (7) follows by integrating by parts, assuming that there are no boundary terms, and using the CauchyRiemann equations.

In order to check for the appearance of boundary terms as in (12), we need the $L_{c}$ evolution of the observables (see below and Appendix B) and the time evolution of the probability density $P$ by solving the FPE with the initial condition (21).

\section{A. Indirect evidence for boundary terms}

In [2] we found numerically for a somewhat different model that the $L_{c}$ evolved observables $\mathcal{O}(x+i y ; t)$ grow in the $y$ direction as an iterated exponential. The same can be seen here, but we will not go into this. This growth makes the appearance of boundary terms already plausible.

In the following we show explicitly that Eq. (7) is numerically satisfied for short times (up to $t \approx 20$ ), choosing $\beta=0.1$.

The $L_{c}$ evolution of an observable $\mathcal{O}$ is defined by the differential equation

$$
\begin{aligned}
\partial_{t} \mathcal{O}_{k}(z ; t) & =L_{c} \mathcal{O}_{k}(z ; t) \quad(t \geq 0), \\
\mathcal{O}_{k}(z ; 0) & =\exp (i k z)
\end{aligned}
$$

with

$$
L_{c}=\left[\partial_{z}-S^{\prime}(z)\right] \partial_{z}
$$

We compare [see (36) for the definition of $F_{\mathcal{O}}$ ]

$$
\int d x d y P(x, y ; 0) \mathcal{O}(x+i y ; t) \equiv F_{\mathcal{O}}(t, t) \equiv\langle\mathcal{O}(t)\rangle_{0}
$$

with

$$
\begin{aligned}
\int d x d y P(x, y ; t) \mathcal{O}(x+i y ; 0) & \equiv\langle\mathcal{O}(0)\rangle_{t} \\
& \equiv\langle\mathcal{O}\rangle_{t} \equiv F_{\mathcal{O}}(t, 0) .
\end{aligned}
$$

Here $P(x, y ; t)$ is the solution of the real Fokker-Planck equation (FPE)

$$
\frac{\partial}{\partial t} P(x, y ; t)=L^{T} P(x, y ; t),
$$

with

$$
L^{T}=\partial_{x}\left[\partial_{x}-K_{x}\right]-\partial_{y} K_{y},
$$

and initial condition (21), which describes the time evolution of the probability density under the CL process.

For our model the FPE is (26) with the drift force (17). Equation (26) is solved numerically as well; some details are found in Appendix C.

Figure 3 compares (24) and (25) for the Fourier modes

$$
\mathcal{O}_{k}(z)=\exp (i k z)
$$

for $k=1,2,3$, Langevin times $t$ between 0 and 50 and $\beta=0.1$.

It is seen that the left-hand side (24) reaches its asymptotic value already for quite short Langevin times (around $t \approx 7$ ). This is in accordance with the value of the smallest nonzero eigenvalue $\lambda_{1} \approx-1$ of $L_{c}$ [cf. Eq. (B6)]. For this value of $\beta$ also the right-hand side does the same; for $t \lesssim 20$ there is no difference visible between the left and the right-hand sides (dashed and solid curves). This indicates that any boundary terms are negligible there.

So there is a "plateau" corresponding to the correct value in the solid curve, and the boundary term starts picking up around $t=20$.

In Fig. 4 we show (in black) the evolution of the first mode up to time $t=200$. It is seen that after the plateau it converges to zero, the value corresponding to the stationary solution (15) of the FPE. We will return to this figure in Sec. V.

\section{B. Direct study of the boundary terms}

We next study explicitly the evolution of the boundary term Eq. (12) for the modes $k=1,2,3$ and $\tau=0$ with

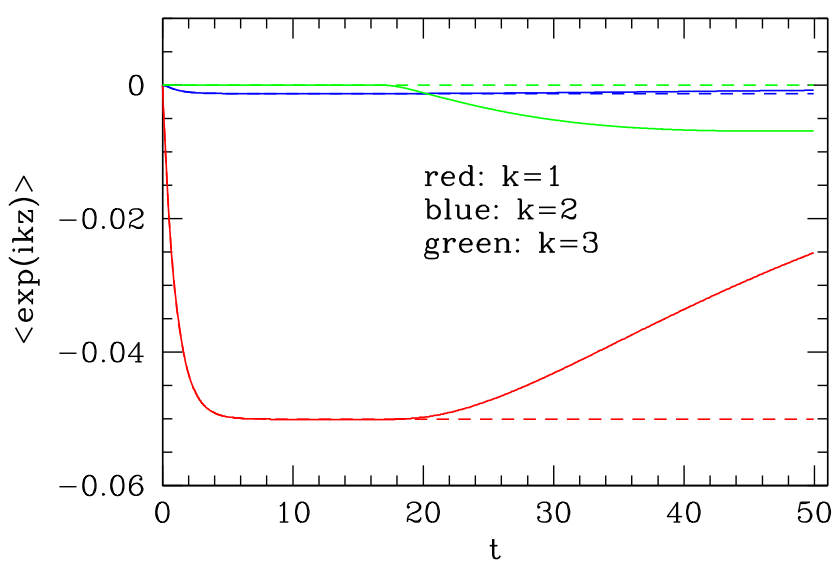

FIG. 3. Comparison of expectation values using the FPE evolution of $P$ (solid lines) Eq. (25) with the $L_{c}$ evolution of the observables [dashed lines-Eq. (24)] for $\beta=0.1$. Note that for times up to about 20 the dashed and solid lines are practically indistinguishable. 


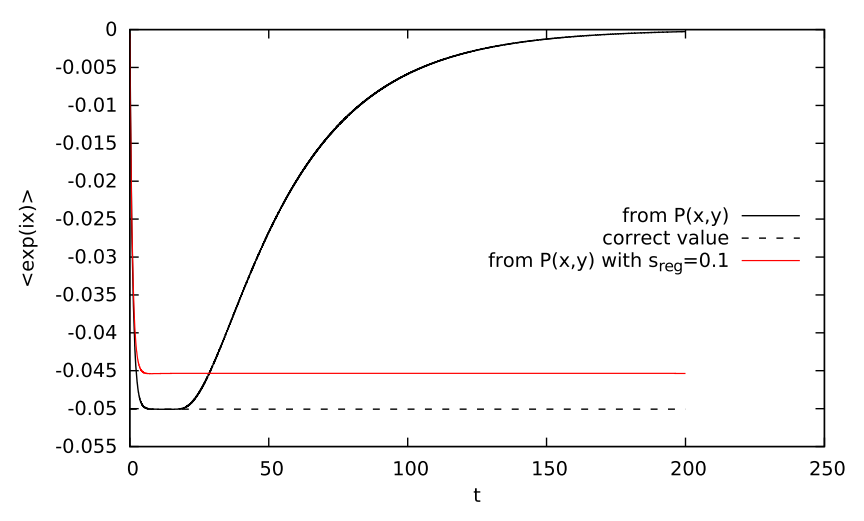

FIG. 4. FPE evolution of $O_{1}$ at $\beta=0.1$ (black). For comparison we show the evolution with regularization $K_{R, y}=-s y, s=0.1$ (red), see Sec. IV.

Langevin time $t$. As explained in Appendix A the definition of this term implies a certain order of limits: Integrate by parts restricted to $|y| \leq Y$, send $t \rightarrow \infty$ and then $Y \rightarrow \infty$ (notice that this does not require a separate simulation but a certain processing of the data). We obtain for the $k$ th mode in our model:

$$
\begin{aligned}
B_{k}(Y ; t, 0)= & \left.\frac{\partial}{\partial \tau} F_{k}(t, \tau)\right|_{\tau=0} \\
= & \beta \int_{-\pi}^{\pi} d x \sin (x) \cosh (Y) \mathrm{e}^{i k x} \\
& \times\left[P(x, Y ; t) \mathrm{e}^{-Y}-P(x,-Y ; t) \mathrm{e}^{Y}\right] .
\end{aligned}
$$

We first note that we can take the limit $t \rightarrow \infty$ of this expression, using the fact that $P(x, y ; t)$ indeed converges to Eq. (15), which was verified before. We obtain

$$
\begin{aligned}
& B_{k}(Y ; \infty, 0) \\
& \quad=-2 \beta \int_{-\pi}^{\pi} d x \frac{\sin (x) \cosh (Y) \mathrm{e}^{i k x} \sinh (Y)}{4 \pi \cosh ^{2}(Y)} .
\end{aligned}
$$

For $k= \pm 1$ this can be evaluated to

$$
B_{\mp 1}(Y ; \infty, 0)=\mp \frac{i \beta}{2} \tanh (Y),
$$

(converging to $\mp i \beta / 2$ for $Y \rightarrow \infty$ ), whereas for $|k|>1$ we obtain 0 .

In Fig. 5 we compare $B_{1}$ determined numerically for Langevin times up to $t=200$ with the asymptotic value at $t=\infty$ for $\beta=0.1$. $Y$ was chosen to be 5 which is close to the asymptotic value $Y=\infty(\tanh (5)=0.99991$.$) . We see$ here directly that the boundary term stays very small up to $t \lesssim 20$, then picks up and approaches the analytically determined value $-i \beta / 2$. For the value $\beta=0.1$ it also follows closely the difference between the first mode shown in Fig. 4 and the correct value, but this cannot remain true for larger $\beta$.
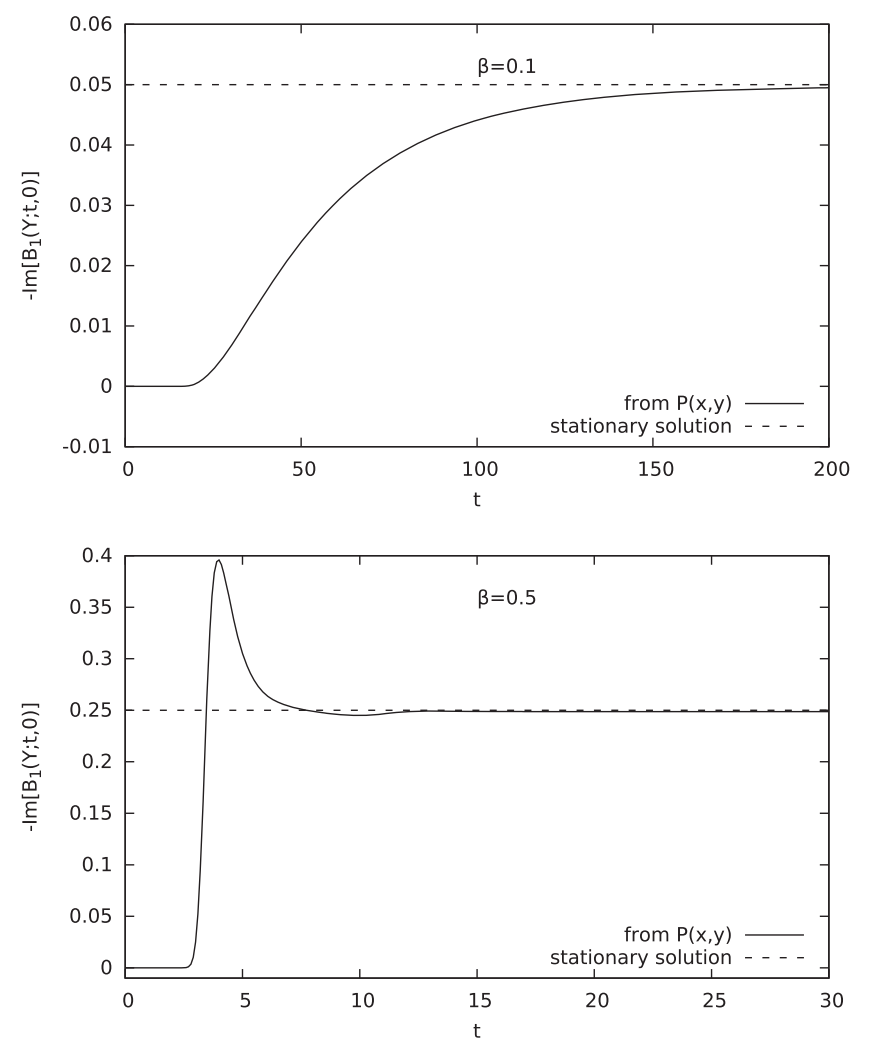

FIG. 5. Numerical evolution via FPE of the imaginary part of the boundary term Eq. (29) for $\beta=0.1$ (top) and $\beta=0.5$ (bottom), $k=1$ and $Y=5$.

We also checked the cases $k=2,3$ and found that $B_{k}$ also starts out very small up to about $t=20$, then increases and for large $t$ seems to go to the asymptotic value 0 determined above. But one has to keep in mind that for $|k| \geq 2$ we are for $Y \rightarrow \infty$ evaluating a conditionally convergent integral; the CL process or equivalently the FPE evaluates that integral in a different way and may therefore produce different results. For $k=1$, however, there is no such subtlety and the boundary term $B_{1}(\infty ; t, 0)$ agrees with the slope of $F_{k}(t, \tau)$ at $\tau=0$.

In Fig. 6 we also show the boundary term $B_{1}$ for different values of the cutoff $Y$, showing the fast approach to the asymptotic value. Note that in the lower panel we show the boundary term as measured using the CLE alone, without making use of the Fokker-Planck evolution, which would be prohibitively costly in a lattice model.

So we established implicitly and explicitly that boundary terms appear appreciably only after some Langevin time. Nonvanishing boundary terms at any $t>0$ invalidates the argument for correctness.

It can also seen by inspection of Eq. (29) that the presence of the observable $\mathcal{O}_{1}$ is essential; the distribution of the drift force alone goes to zero. Quite generally it is the product of observable, drift and probability $P$ that decides about the presence or absence of boundary terms. 

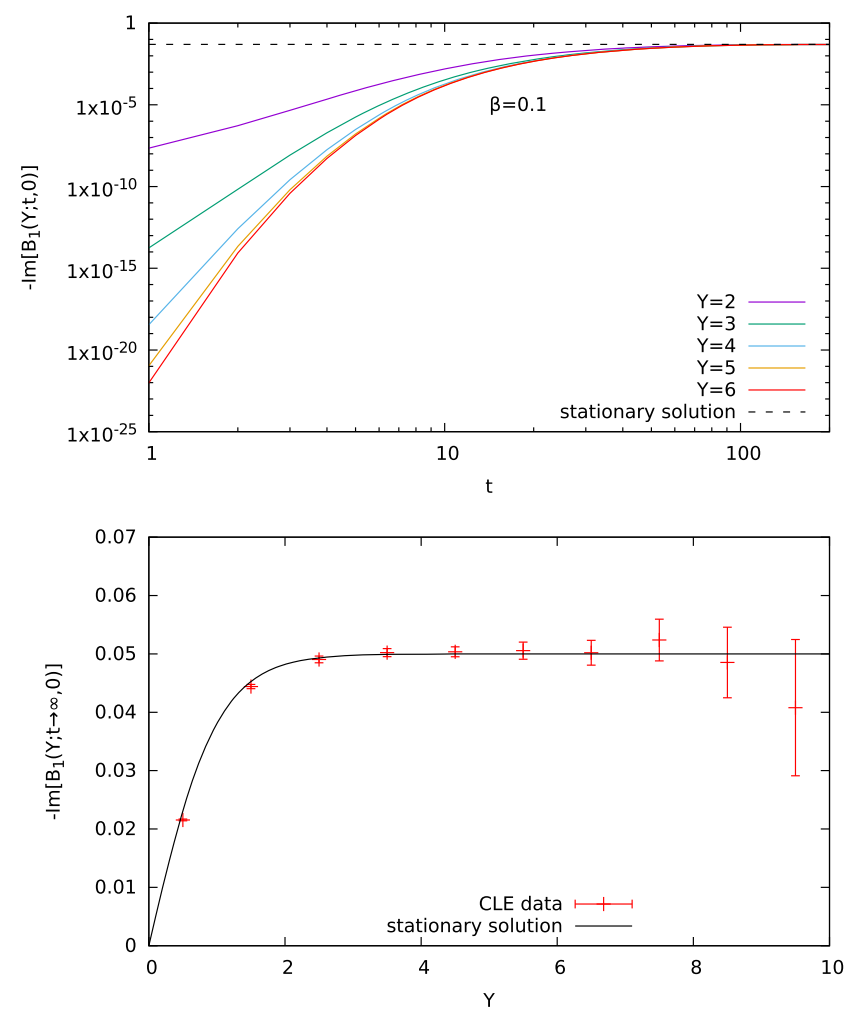

FIG. 6. Top panel: numerical evolution via FPE of the imaginary part of the boundary term Eq. (29) for $k=1, \beta=0.1$ and different values of $Y$ vs $t$. Bottom panel: the same boundary term evaluated via Langevin simulation at asymptotic $t$ vs $Y$.

\section{Boundary terms and skirts}

Thinking now of $Y$ not as a cutoff, but as a variable, and denoting it by $y$ again, we see that the first term of the boundary term $B_{1}(y ; \infty, 0)$, considered as a function of $y$ Eq. (29) is just the probability density of the observable

$$
v(y) \equiv \operatorname{Im} \int d x K_{y}(x, y) \mathcal{O}_{1}(x-i y) \sim \mathrm{e}^{2 y}
$$

for large $y$. The nonvanishing of the boundary term is the fact that

$$
\lim _{y \rightarrow \infty} v(y) P_{y}(y) \neq 0 .
$$

On the other hand the distributions of $v$ itself has a density $p(v)$, related to $P$ by

$$
p(v)=P_{y}(y(v)) \frac{d y}{d v} .
$$

This can easily be worked out, but the point is that for large $y$

$$
p(v) \sim v^{-2},
$$

which shows that there is no finite expectation value of $v$ since $v p(v)$ is not integrable.

In other words: $a$ "skirt" in the distribution of $K_{y} \mathcal{O}$ falling off like the power -2 or more slowly corresponds to a nonvanishing (possibly diverging) boundary term. Note, however, such a simple reasoning is only possible because here $P$ is independent of $x$.

\section{The interpolating function}

So far we have only compared $F_{k}(t, 0)$ and $F_{k}(t, t)$. But it is instructive also to look at the interpolating function $F_{k}(t, \tau)$

$$
F_{\mathcal{O}}(t, \tau) \equiv \int P(x, y ; t-\tau) \mathcal{O}(x+i y ; \tau) d x d y,
$$

which should be independent of $\tau$ for the correctness argument to hold. This is shown in Fig. 7 for $k=1$ for $\beta=0.1$ and $\beta=0.5$.

Again it is seen that for $\beta=0.1, t \lesssim 20$ the curves are flat, indicating the absence of any appreciable boundary terms. For $t>20$ a $\tau$ dependence develops, being maximal near $\tau=0$. This is understandable from what we have seen: the FPE evolution of $P$ proceeds up to time $t-\tau$, which allows for the boundary terms to arise. On the other hand, for $\tau \gtrsim 7 \mathcal{O}_{k}(z ; \tau)$ has practically reached its asymptotic limit (cf. Appendix B), in which only the constant mode survives; this constant can be pulled outside the integral defining $F$, so that for $t, \tau>7$

$$
\mathcal{O}_{k}(z ; \tau) \approx \frac{1}{2 \pi} \int_{-\pi}^{\pi} d x^{\prime} \rho\left(x^{\prime}\right) \mathcal{O}_{k}\left(x^{\prime}\right)=\left\langle\mathcal{O}_{k}\right\rangle_{c}
$$

and

$$
F_{k}(t, \tau) \approx \frac{1}{2 \pi} \int d x d y P(x, y ; t)\left\langle\mathcal{O}_{k}\right\rangle_{c}=\left\langle\mathcal{O}_{k}\right\rangle_{c} .
$$

i.e., the correct value (where we used the fact that the density $P$ is always normalized).

At small $t, \tau$ flat curves for $F_{1}(t, \tau)$ indicate that CL gives the correct values, however these are dependent on the initial condition if the process did not yet thermalize. This is seen in Fig. 7, bottom plot, for $\beta=0.5$.

Notice that the slope of $F_{k}(t, \tau)$ appears maximal near $\tau=0$ for large $t$. Therefore the estimation of noxious boundary terms as defined in (29) is relevant for judging the asymptotic correctness of the CL procedure-cf. Figs. 5 and 7.

Plots similar to Fig. 7 appeared in [2] for a different model.

\section{E. Evolution of some marginal distributions}

For $\beta=0.1$ we saw clearly the evolution first apparently converging to the correct value and then departing from it 

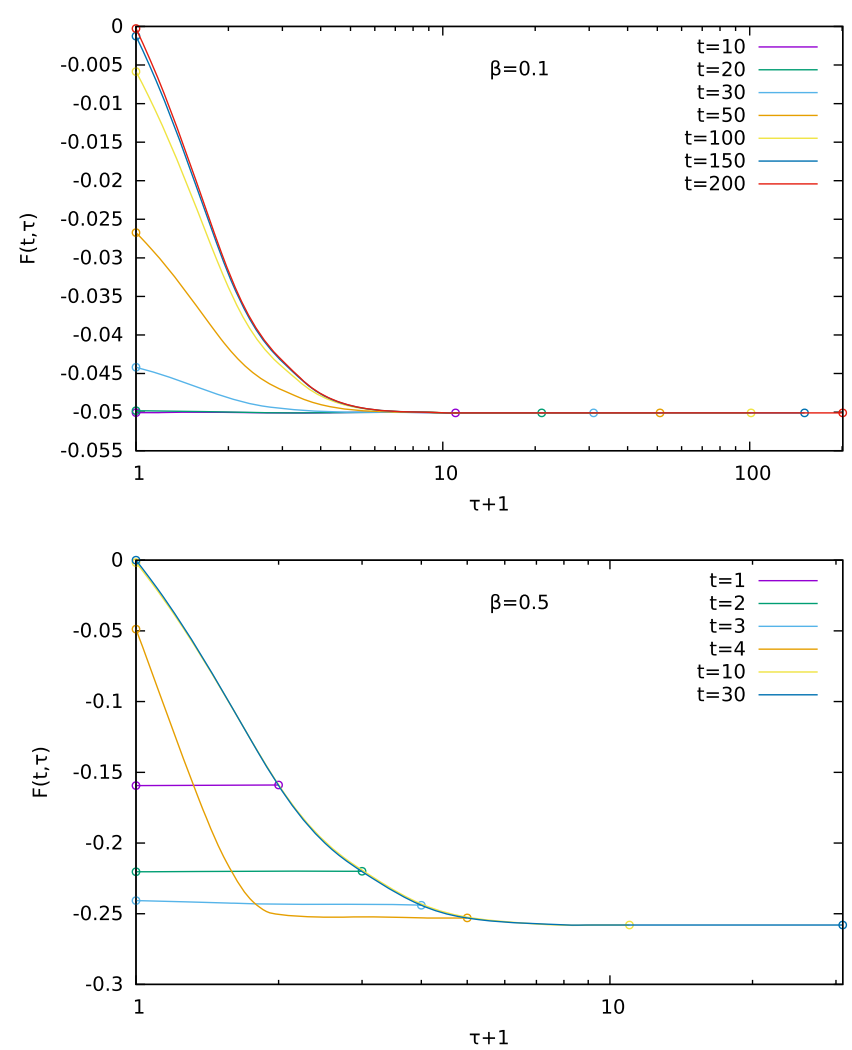

FIG. 7. The interpolating function $F_{1}(t, \tau)$ defined in (36) for the first mode; $\beta=0.1$ (top) and $\beta=0.5$ (bottom) for various values of $t$; the small circles denote the beginning and end of the respective curves.

(the plateau in Fig. 3). Similar behavior in Langevin time was observed in a real time $S U(2)$ lattice simulation [11]. This is also reflected in some marginal distributions.

In Fig. 8 we show the evolution of $P_{x}(x ; t)=$ $\int d y P(x, y ; t)$ for $\beta=0.1$. It starts out flat, corresponding to our choice of initial condition; at $t=10$ and $t=20$ it shows maximal structure, while for larger $t$ it approaches a flat distribution again, in agreement with (15).

The distribution of the first mode also show a similar behavior. Of interest is the imaginary part. Its density is

$$
\sigma(u ; t) \equiv \int d x d y P(x, y ; t) \delta\left(\sin (x) e^{-y}-u\right)
$$

We present in Fig. 9 histograms for $\sigma(u ; t)$, obtained from the numerical solution of the FPE; for the limiting distribution $P(x, y ; \infty)=1 /\left(4 \pi \cosh ^{2}(y)\right.$ we can evaluate (39) analytically:

$$
\begin{aligned}
\sigma(u ; \infty) & =\frac{|u|}{\pi} \int_{-1}^{1} d t \frac{t^{2}}{\left(t^{2}+u^{2}\right)^{2} \sqrt{1-t^{2}}} \\
& =\frac{1}{2\left(1+u^{2}\right)^{3 / 2}} .
\end{aligned}
$$

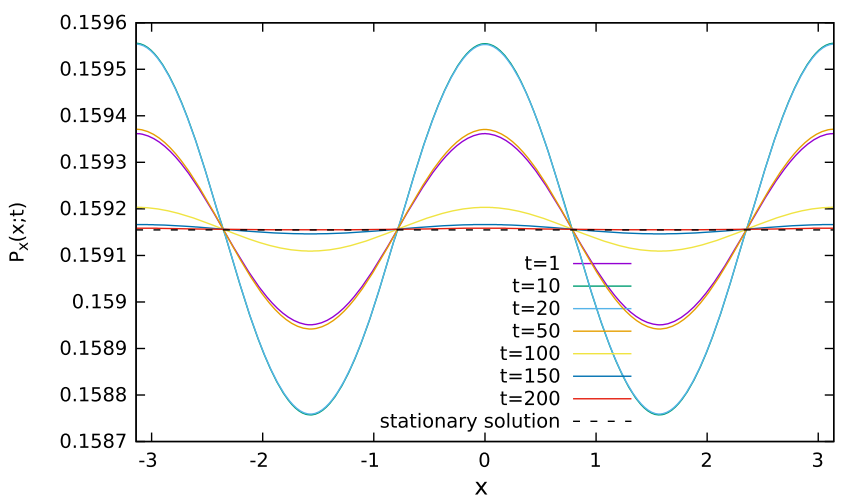

FIG. 8. The marginal distribution $P_{x}(x ; t)$ obtained from solving the Fokker-Planck equation for $\beta=0.1$.

Figure 9 shows first the development of an asymmetric structure with two maxima, whereas for larger $t$ one sees clearly the approach to the symmetric analytic result (40).

In this context it may be of interest to compare with the criterion of [13]. In Fig. 10 we show the distribution of the drift itself for various Langevin times in double logarithmic scale. The decay always seems powerlike, albeit with a very high power for short times. This would indicate, according
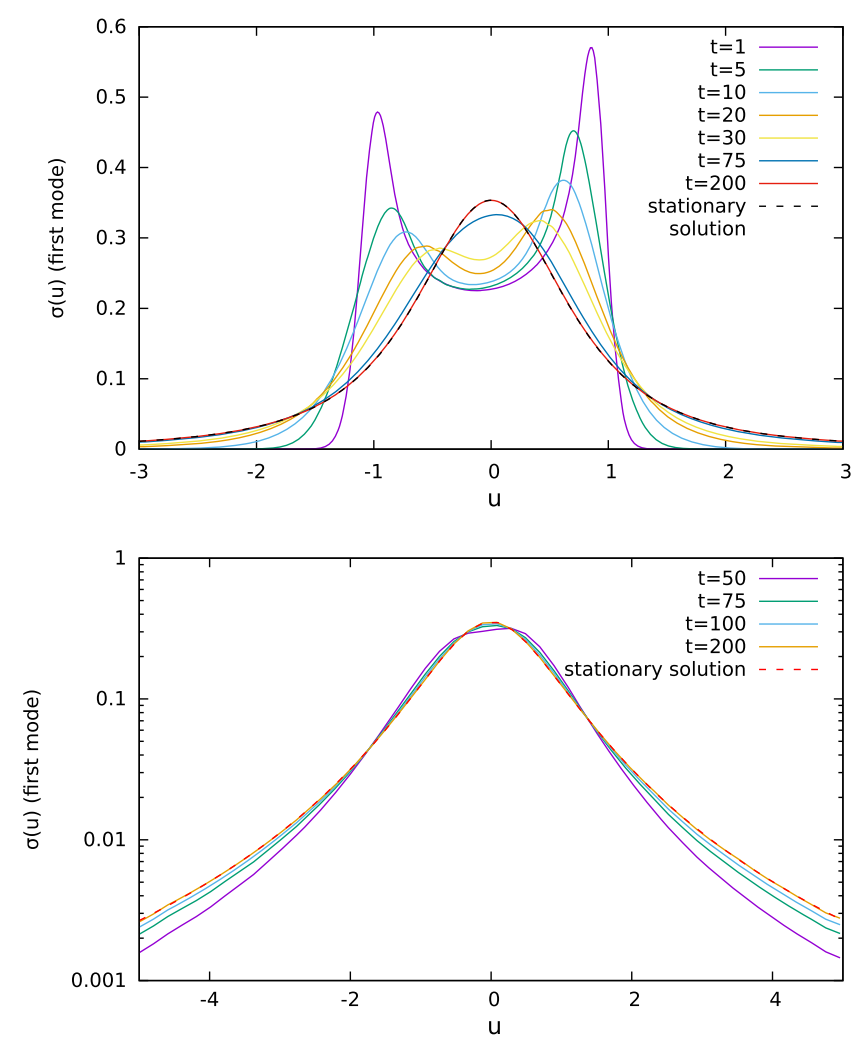

FIG. 9. Evolution of the distribution of the first mode $\sigma(u)$. Top panel: times from $t=1$ to 200 in linear scale; bottom panel: times from $t=50$ to 200 in $\log$ scale. Again note that for $t=200$ no difference is visible between the numerical results and the analytic expression. 


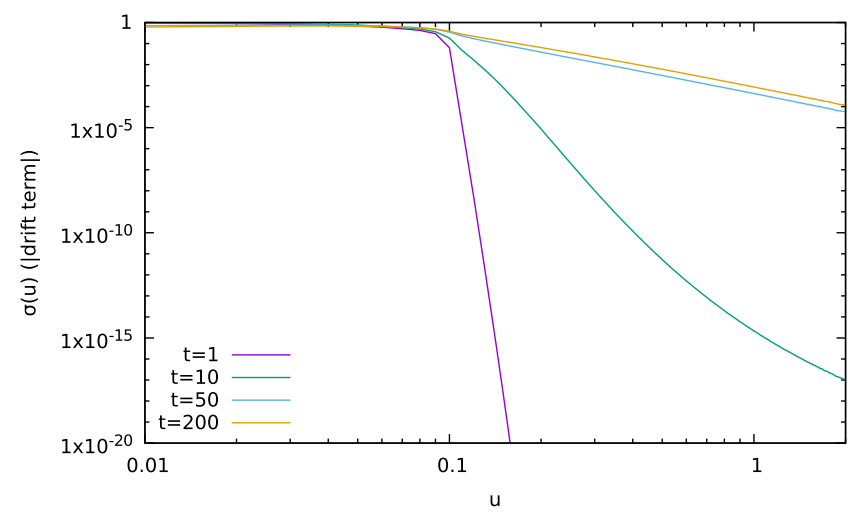

FIG. 10. Histograms of the drift for different Langevin times $t$ and $\beta=0.1$.

to [13], that even for the small times where the CL results seem to be correct (but not necessarily converged) there might be a tiny boundary term making the results incorrect by an invisible amount.

\section{ILLUSTRATION OF THE EFFECT OF THE BOUNDARY TERMS IN A REGULARIZED MODEL}

In the preceding section we described how the boundary terms accumulate in the Langevin (and Fokker-Planck) evolution, spoiling the proof of convergence such that the process would lead to wrong results.

Here we want to explicitly see the effect of those terms by considering a "regularization" of the model using a damping term in the action, $S_{R}=\frac{s}{2} x^{2}$, which leads to a modification of the drift by $K_{R}(z)=-s z$ (a similar regularization has been used in [14]; we thank J. Drut and A. C. Loheac for making us aware of this). The philosophy of this regularization is very similar to that of dynamical stabilization [15]. In both cases, and different from modifications using symmetries, such as in the gauge cooling paradigm, the dynamics is really changed, but in a way intended to be controllable.

For $s=0$ we regain the original model Eq. (13) (including its problems) while for $s>0$ we should observe an interplay between the original tendency to build boundary terms and their damping in the modified model, allowing us to estimate the effect of these terms. This particular modification leads to loss of periodicity in $x$ which becomes noncompact at $s>0$. The CLE process was allowed to drift unbounded in the full $z$ plane and the exact integral was correspondingly done in the infinite interval. The following plots show $O_{1}=\operatorname{Im}\left\langle e^{i z}\right\rangle$. We see from Fig. 11 that the regularization stabilizes the expectation values in the CLE evolution. When the nonregularized data show a plateau at the correct value for intermediary $t$ the regularization extends this plateau into the asymptotic region ( $\beta=0.1$ case). When a plateau is
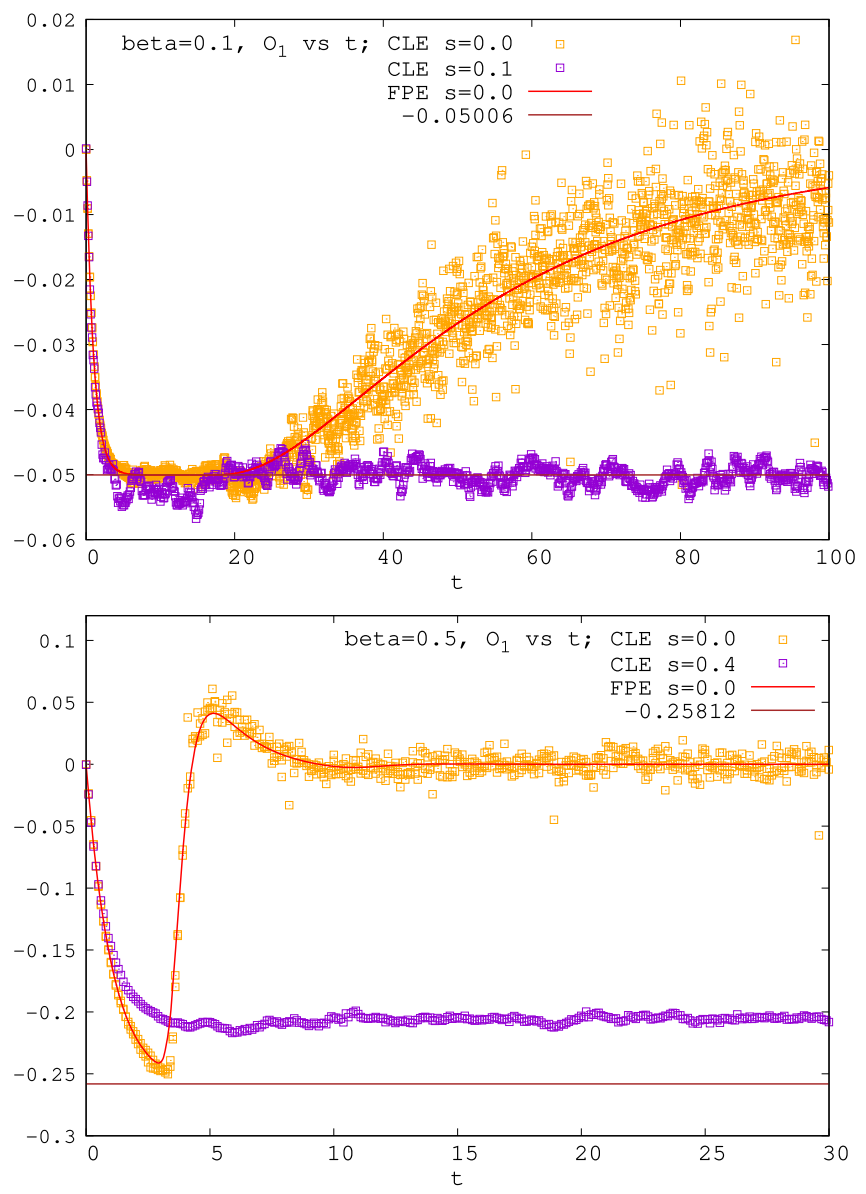

FIG. 11. Comparison of the $O_{1}$ expectation values from FPE (solid line) and CLE and from regularized CLE, vs $t$. Top: $\beta=0.1, s=0$ and 0.1 , respectively. Bottom: $\beta=0.5, s=0$ and 0.4 , respectively.

missing the regularization still stabilizes the expectation value $(\mathrm{EV})$ but at a value shifted from the correct one ( $\beta=0.5$ case), since now a larger $s$ is needed to counteract the boundary terms.

Note that an alternative regularization of the process itself is to modify only the imaginary drift by a damping term $K_{R, y}=-s y$. This leads to similar results (see Fig. 4, here from the FPE evolution), and has the advantage that periodicity in $x$ is preserved. We preferred the action variant, however, also since it allows us to obtain exact correct results for the regularized model by simple numerical integration.

In Fig. 12 we show the $s$ dependence in CLE for the regularized model for the same values of $\beta$. The plots suggest an extrapolation toward the exact expectation value (EV) for $s \rightarrow 0$, however this might not be simply linear, but depend on the particular regularization, $\beta$, etc. Therefore we mean this discussion not yet as a direct cure but mainly as illustration of the effects of the boundary terms on the EV's. For $k=1$, e.g., these effects can be estimated from the distance between the CLE regularized 

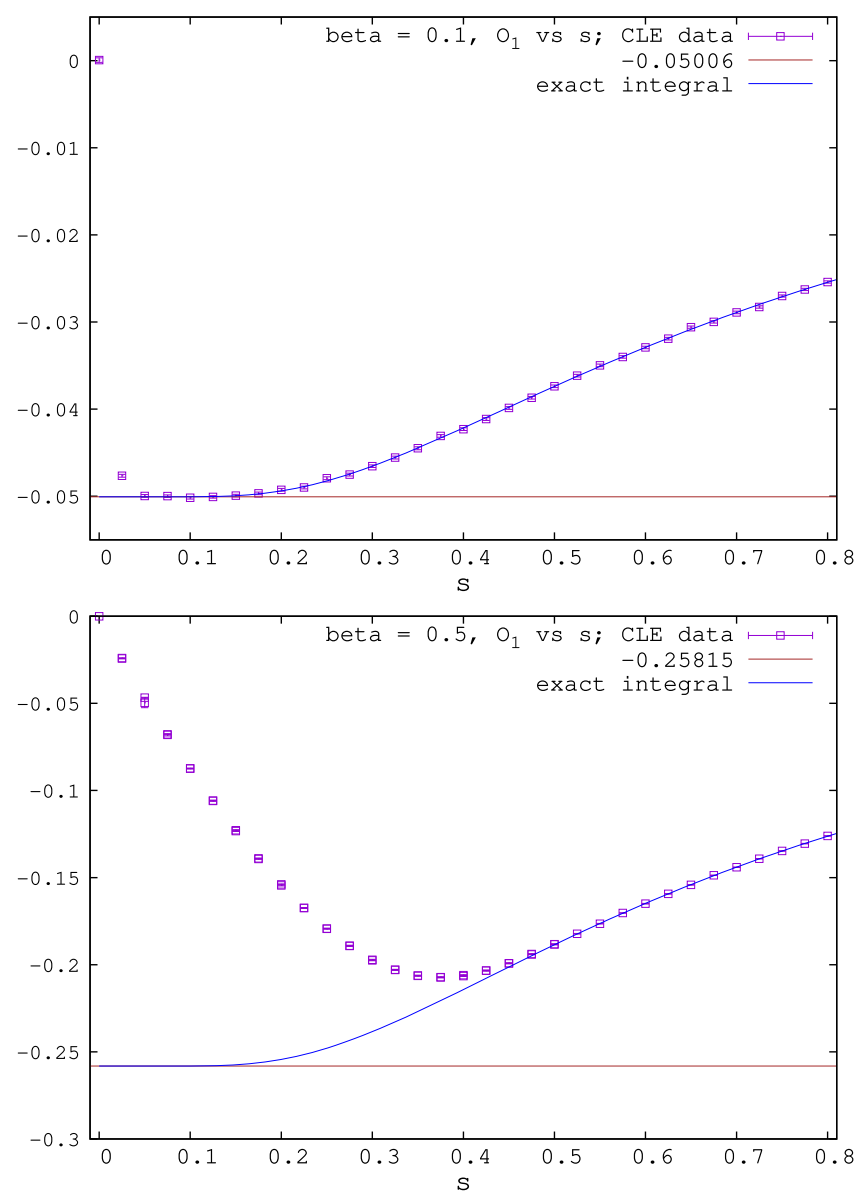

FIG. 12. Dependence of $O_{1}$ on the regularization parameter $s$ of the CLE simulation at $\beta=0.1$ (top) and 0.5 (bottom). The solid lines show the exact correct values from numerical integration.

data and the exact values from the numerical integration of the regularized model: As can be seen from the figure at small $s$ these effects are still present while gradually vanishing with increasing $s$.

Finally it is again instructive to look at the histograms of the drift itself, as advocated by [13], to see the effect of the regularization. This is shown in Fig. 13. One can see that the distribution seems to show powerlike decay $s=0$ whereas for $s=0.1$ the decay appears to be exponential. This supports the criterion of [13], because for the value of $\beta=0.1$ used here, already $s=0.1$ suffices to bring the CL results into agreement with the correct results of the regularized as well as the unregularized model, which are indistinguishable in this case, as shown in Fig. 12.

\section{CONCLUSIONS}

We have in great detail analyzed a simple example in which the CL fails, establishing very explicitly that the failure is due to boundary terms spoiling the correctness argument, as argued already long ago [1,2]. The absence of

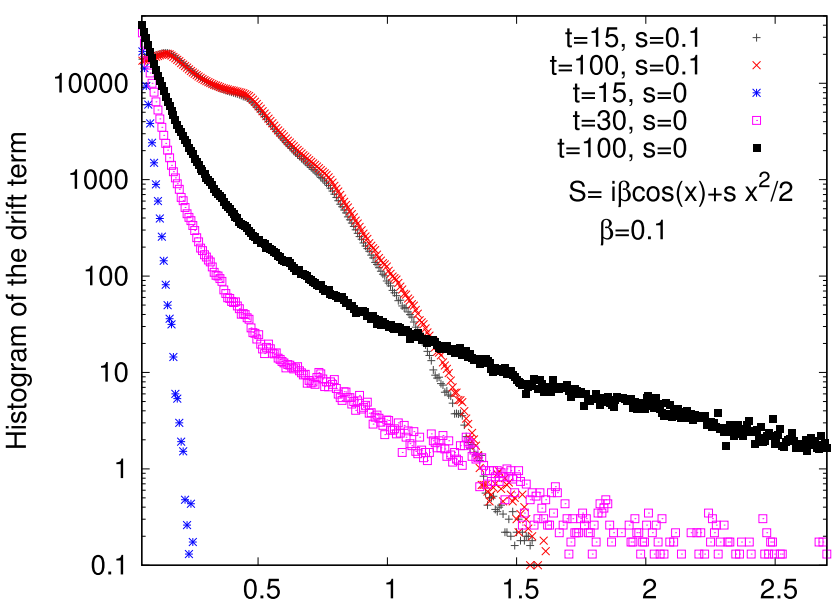

FIG. 13. Histogram of the drift for $\beta=0.1$ and various Langevin times, comparing $s=0$ and $s=0.1$.

such boundary terms requires that the product of observable, drift force and probability distribution $(\mathcal{O} K P)$ goes to zero in the noncompact (imaginary) directions. The relation between boundary terms and skirts, i.e., decay of distributions was addressed in Subsection III C, making clear that possible skirts in the distribution of the product $\mathcal{O} K P$ and not just $K P$ are relevant.

Generally the $\tau$-dependent boundary term (A16) cannot be estimated in a realistic (lattice) calculation. Fortunately, however, the considerations in this paper suggest that relevant for the correctness of the asymptotic (large $t$ ) EV's is the boundary term at $\tau=0, B_{k}(t, 0)$, as defined in (29). This term appears to approximately maximize $B_{k}(t, \tau)$ and it stabilizes at large $t$; it is accessible in principle to online monitoring using the CLE alone, and may provide a correctness criterion for the EV's obtained in the CL simulation.

As remarked before, uncovering the boundary term requires a certain processing of the data obtained in the simulation, implying essentially sampling first at a fixed value of a quantity specifying the boundary in the noncompact directions (in a lattice gauge theory for instance the unitarity norm or some other related quantity) before taking the other limits. This however does not require a separate simulation.

\section{ACKNOWLEDGMENTS}

M.S., E.S. and I.-O.S. gratefully acknowledge kind support from DFG under Grant No. Sta 283/16-2. D. S. gratefully acknowledges funding by the DFG grant Heisenberg Programme (SE 2466/1-2). The authors acknowledge support by the High Performance and Cloud Computing Group at the Zentrum für Datenverarbeitung of the University of Tübingen, the state of Baden-Württemberg through bwHPC and the German Research Foundation (DFG) through Grant No. INST 37/935-1 FUGG. 


\section{APPENDIX A: THE ARGUMENT FOR CORRECTNESS REVISITED}

We briefly revisit the formal proof of correctness for the CL method for our simple periodic one-dimensional models, spelling out the conditions needed for it to work as well as the mechanisms that may lead either to no convergence or "wrong convergence" of the CL process (cf. $[2,4,5])$.

$P(x, y ; t)$ is the time dependent probability distribution corresponding to the $\mathrm{CL}$ process, determined by the real Fokker-Planck equation (26).

We also consider the time evolution of the complex density $\rho(x ; t)$ by the complex Fokker-Planck equation

$$
\frac{\partial}{\partial t} \rho(x ; t)=L_{c}^{T} \rho(x ; t),
$$

where now the complex Fokker-Planck operator $L_{c}^{T}$ is

$$
L_{c}^{T}=\partial_{x}\left[\partial_{x}+S^{\prime}(x)\right] .
$$

The initial conditions for (26) and (A1) are required to be consistent, i.e.,

$$
\begin{aligned}
P(x, y ; 0) & =\rho(x ; 0) \delta(y) \geq 0 \\
\int \rho(x ; 0) d x & =1 .
\end{aligned}
$$

The crucial point is that one can now, under conditions to be spelled out below, show that

$$
\int d x \mathcal{O}(x) \rho(x ; t)=\int d x d y \mathcal{O}(x+i y) P(x, y ; t) .
$$

If in addition the operator $L_{c}^{T}$ has spectrum in the left half plane with 0 a nondegenerate eigenvalue, if follows that

$$
\lim _{t \rightarrow \infty} \int d x \mathcal{O}(x) \rho(x ; t)=\int d x \mathcal{O}(x) \rho(x)
$$

and by (A4)

$$
\begin{aligned}
& \lim _{t \rightarrow \infty} \int d x d y \mathcal{O}(x+i y) P(x, y ; t) \\
& =\int d x \mathcal{O}(x) \rho(x)
\end{aligned}
$$

By our choice of initial conditions, (A4) holds for $t=0$. For $t>0$ we consider $F_{\mathcal{O}}(t, \tau)$ defined in Eq. (36), which interpolates between the two sides of (A4):

$$
F_{\mathcal{O}}(t, \tau) \equiv \int P(x, y ; t-\tau) \mathcal{O}(x+i y ; \tau) d x d y,
$$

with $\mathcal{O}(x+i y ; t)$ defined by solving the differential equation (22) $L_{c}$, the complex Langevin operator, is the transpose of $L_{c}^{T}$ :

$$
L_{c}=\left[\partial_{z}-S^{\prime}(z)\right] \partial_{z}
$$

We call the solution of Eq. (22) the " $L_{c}$ evolved" observable.

The interpolating property follows from

$$
\begin{aligned}
& F_{\mathcal{O}}(t, 0)=\int d x d y \mathcal{O}(x+i y) P(x, y ; t)=\langle\mathcal{O}\rangle_{t} \\
& F_{\mathcal{O}}(t, t)=\int d x \mathcal{O}(x, t) \rho(x ; 0)=\langle\mathcal{O}(t)\rangle_{c},
\end{aligned}
$$

see Sec. III A, Eqs. (24) and (25). The first equality is obvious, the second one follows by integration by parts in $x$; because of periodicity there are no boundary terms. (A4) would follow if we could prove

$$
\begin{aligned}
\frac{\partial}{\partial \tau} F_{\mathcal{O}}(t, \tau)= & -\int\left(L^{T} P(x, y ; t-\tau)\right) \mathcal{O}(x+i y ; \tau) d x d y \\
& +\int P(x, y ; t-\tau) L_{c} \mathcal{O}(x+i y ; \tau) d x d y=0 .
\end{aligned}
$$

This would again follow from integration by parts, provided there are no boundary terms. For the term $\partial_{x}^{2}$ of both $L^{T}$ and $L_{c}$ this is obvious because of periodicity, so we can drop these terms, obtaining

$$
\begin{aligned}
\frac{\partial}{\partial \tau} F_{\mathcal{O}}(t, \tau) & =\int \mathcal{O}(x+i y ; \tau)\left(\partial_{x} K_{x}+\partial_{y} K_{y}\right) P(x, y ; t-\tau) d x d y \\
& -\int P(x, y ; t-\tau) S^{\prime}(x+i y) \partial_{x} \mathcal{O}(x+i y ; \tau) d x d y .
\end{aligned}
$$

In [1] we argued that $\mathcal{O}(x+i y ; \tau)$ is holomorphic for any $\tau$, i.e., it obeys the Cauchy Riemann equations

$$
\partial_{y} \mathcal{O}(x+i y ; \tau)=i \partial_{x} \mathcal{O}(x+i y ; \tau) .
$$

This allows us to write the second term of the right-hand side of (A12) as

$\int P(x, y ; t-\tau)\left(K_{x} \partial_{x}+K_{y} \partial_{y}\right) \mathcal{O}(x+i y ; \tau) d x d y$.

Again the part involving $\partial_{x}$ can be canceled against the corresponding term in the first term of (A14) using integration by parts in $x$, so we only have to consider 


$$
\begin{aligned}
\frac{\partial}{\partial \tau} F_{\mathcal{O}}(t, \tau)= & \int\left(\partial_{y} K_{y} P(x, y ; t-\tau)\right) \mathcal{O}(x+i y ; \tau) d x d y \\
& +\int P(x, y ; t-\tau) K_{y} \partial_{y} \mathcal{O}(x+i y ; \tau) d x d y .
\end{aligned}
$$

We have to interpret this as a the limit $Y \rightarrow \infty$ of the integral restricted to $|y| \leq Y$. For finite $Y$ (A15), since the integrand is a total derivative, this is given by the boundary term

$$
\begin{aligned}
B_{\mathcal{O}}(Y ; t, \tau) \equiv & \int\left[K_{y}(x, Y) P(x, Y ; t-\tau) \mathcal{O}(x+i Y ; \tau)\right. \\
& \left.-K_{y}(x,-Y) P(x,-Y ; t-\tau) \mathcal{O}(x-i Y ; \tau)\right] d x .
\end{aligned}
$$

Evaluating this term at $\tau=0$ leads then for our model to (29) where we can then take the large $t$ limit to obtain for our model, obtaining (30). This form of the boundary term makes clear that correctness requires sufficient decay of the products $K_{y} P \mathcal{O}$.

Notice that if we take the $t \rightarrow \infty$ limit directly in (A11) the first term vanishes by stationarity and the second one leads to the "correctness conditions" (CC) defined in [2] and is approximately zero by stationarity. Hence, it might appear that the boundary term vanishes and we might erroneously conclude correctness of the results. Therefore the $\mathrm{CC}$, while expressing convergence and being necessary for correctness, are not sufficient.

\section{APPENDIX B: THE CORRECT EVOLUTION}

What was called the "correct time evolution" $\langle\mathcal{O}(t)\rangle_{0}$ of the expectation value of $\mathcal{O}$ is simply the expectation value in the starting probability density $P(x, y ; 0)$ of the $L_{c}$ evolved observable $\mathcal{O}$, see Eq. (24). To analyze this we rewrite the Langevin operator $L_{c}$ in the basis of Fourier modes:

$$
\begin{aligned}
L_{c} \exp (i k x)= & -k^{2} \exp (i k x)-\frac{i \beta}{2} k \exp (i(k+1) x) \\
& +\frac{i \beta}{2} k \exp (i(k-1) x)
\end{aligned}
$$

or equivalently, for a general observable

$$
\begin{aligned}
\mathcal{O}(x) & =\sum_{k} a_{k} \exp (i k x) \\
\left(L_{c} a\right)_{k} & =-k^{2} a_{k}-\frac{i \beta}{2}(k-1) a_{k-1}+\frac{i \beta}{2}(k+1) a_{k+1} .
\end{aligned}
$$

So $L_{c}$ is represented on the Fourier coefficients by the sparse infinite matrix with elements

$$
\left(L_{c}\right)_{k l}=-k^{2} \delta_{k l}-\frac{i \beta}{2}(k-1) \delta_{k-1, l}+\frac{i \beta}{2}(k+1) \delta_{k+1, l} .
$$

It is easy to compute numerically the action of $\exp \left(t L_{c}\right)$ on observables of the form $\mathcal{O}_{k}=\exp (i k x)$; cutting off the modes at $|k| \geq K$ with $K=50$ and $K=150$, and for $t=50$, gave identical results, with only the constant mode surviving. Its value agrees to at least 5 digits with

$$
\lim _{t \rightarrow \infty} \exp \left(t L_{c}\right) \mathcal{O}_{k}=\int d x \rho(x) \mathcal{O}_{k}(x)
$$

i.e., the correct expectation value.

We also checked, using Mathematica, that the eigenvalues of the truncated matrix $\left(L_{c}\right)_{k l}$ have negative real part except for the unique zero eigenvalue corresponding to $a_{k} \propto \delta_{k 0}$. All nonzero eigenvalues are real and doubly degenerate. The one with the smallest modulus determines the approach to the infinite time limit; it depends only weakly on $\beta$, e.g.,

$$
\begin{aligned}
& \lambda_{1}=-0.998333(\beta=0.1) ; \\
& \lambda_{1}=-0.832189(\beta=1)
\end{aligned}
$$

Remark: It is easy to show that by a similarity transformation $L_{c}$ can be transformed into the dissipative operator

$$
\begin{aligned}
-H & =\exp (S / 2) L \exp (-S / 2) \\
& =\frac{d^{2}}{d x^{2}}-\beta^{2} \sin ^{2}(x)-\frac{i \beta}{2} \cos (x) .
\end{aligned}
$$

Dissipativity means $-H-H^{*} \leq 0$, which is obvious. For such operators general theorems guarantee that the spectrum is contained in the left half of the complex plane (see for instance [16]). It is also not hard to see that there is exactly one vector with eigenvalue zero.

\section{APPENDIX C: REMARKS ON THE NUMERICAL SOLUTION OF THE FPE}

The real Fokker-Planck equation in our case is

$$
\begin{aligned}
\frac{\partial P(x, y ; t)}{\partial t}= & {\left[\partial_{x}\left(\partial_{x}-K_{x}\right)-\partial_{y} K_{y}\right] P(x, y ; t) } \\
= & {\left[\partial_{x}^{2}+\beta\left(-2 \sin x \sinh y+\cos x \sinh y \partial_{x}\right.\right.} \\
& \left.\left.-\sin x \cosh y \partial_{y}\right)\right] P(x, y ; t) .
\end{aligned}
$$

Discretizing (C1) in $x$ and $y$ using symmetric derivatives yields 


$$
\begin{aligned}
P(x, y ; t+d t)= & \frac{1}{d x^{2}}\left(P\left(x_{+}, y ; t\right)-2 P(x, y ; t)+P\left(x_{-}, y ; t\right)\right) \\
& -2 \beta \sin x \sinh y P(x, y ; t) \\
& +\frac{\beta}{2 d x} \cos x \sinh y\left(P\left(x_{+}, y ; t\right)-P\left(x_{-}, y ; t\right)\right) \\
& -\frac{\beta}{2 d y} \sin x \cosh y\left(P\left(x, y_{+} ; t\right)-P\left(x, y_{-} ; t\right)\right),
\end{aligned}
$$

where we defined $x_{ \pm}=x \pm d x$ and similarly for $y$. In case of a regularization term in the $y$-drift $K_{y} \rightarrow K_{y}-s_{y} y$ (see Sec. IV), additional terms occur

$$
\begin{aligned}
P(x, y ; t+d t) \rightarrow & P(x, y ; t+d t)+s_{y} P(x, y ; t) \\
& +s_{y} \frac{y}{2 d y}\left(P\left(x, y_{+} ; t\right)-P\left(x, y_{-} ; t\right)\right) .
\end{aligned}
$$

We solved the Fokker-Planck equation on an $x-y$-grid with parameters $d t=10^{-6}, d x=0.005=d y$, a cutoff in $y$-direction of $Y=5$ was found to be sufficient [compare $(30), \tanh (5) \approx \tanh (\infty)]$, and a cutoff in $x$-direction of $X=3.14$, which is due to the $2 \pi$ periodicity of the problem. Boundary conditions in $x$ and $y$ were both chosen to be periodic. Initial condition were chosen according to (21), however the $\delta$-function was smeared out slightly to avoid numerical issues; so we actually used

$$
P(x, y ; 0)=\frac{1}{2 \pi \sqrt{2 \pi \sigma_{y}^{2}}} e^{-\frac{y^{2}}{2 \sigma_{y}^{2}}},
$$

where we chose $\sigma_{y}=0.1$. Note that using this discretization it is hard to resolve the higher modes. This can be done more easily when solving the Fokker-Planck equation in Fourier space, where it is given by

$$
\begin{aligned}
P(k, y ; t+d t)= & -k^{2} P(k, y ; t) \\
& -\frac{i \beta}{2} \sinh (y)\left(k_{-} P\left(k_{+}, y ; t\right)+k_{+} P\left(k_{-}, y ; t\right)\right) \\
& +\frac{i \beta}{4 d y} \cosh (y)\left(P\left(k_{+}, y_{+} ; t\right)-P\left(k_{-}, y_{+} ; t\right)\right) \\
& +\frac{i \beta}{4 d y} \cosh (y)\left(-P\left(k_{+}, y_{-} ; t\right)+P\left(k_{-}, y_{-} ; t\right)\right),
\end{aligned}
$$

where $k_{ \pm}=k \pm 1$ and similarly for $y$. Here we chose $d t=0.5 \times 10^{-5}, k \in\{-19, \ldots, 20\}, d y=\sqrt{d t}, \quad Y \approx 2.8$ with antiperiodic boundary conditions in $k$ for the imaginary part of $P(k, y ; t)$ and periodic boundary conditions for the real part in $k$ and for $y$. After $t \sim 30$ or so the result strongly depends on the choice of discretization. Hence, we use the $k-y$ discretization to resolve the plateaus in the higher modes and the $x-y$-discretization for everything else.

The solution to the Fokker-Planck equation shows that for $\beta=0.1$ the evolution initially follows the correct evolution. This is suggested by Figs. 7 and 5. By looking at $P(x, y ; t)$ in Figs. 2 and 8 and the histogram of the first mode in Fig. 9, one can see that initially nontrivial structures occur. Those die out and everything approaches the asymptotic solution, which yields the wrong results. This strengthens the argument that until $t \sim 20$ or so CLE yields the correct solution but then the occurrence of boundary terms leads to wrong convergence.
[1] G. Aarts, E. Seiler, and I. O. Stamatescu, Phys. Rev. D 81, 054508 (2010).

[2] G. Aarts, F. A. James, E. Seiler, and I. O. Stamatescu, Eur. Phys. J. C 71, 1756 (2011).

[3] J. Nishimura and S. Shimasaki, Phys. Rev. D 92, 011501 (2015).

[4] G. Aarts, E. Seiler, D. Sexty, and I. O. Stamatescu, J. High Energy Phys. 05 (2017) 044; 01 (2018) 128(E).

[5] E. Seiler, EPJ Web Conf. 175, 01019 (2018).

[6] L. L. Salcedo, Phys. Rev. D 94, 114505 (2016).

[7] A. Mollgaard and K. Splittorff, Phys. Rev. D 88, 116007 (2013).

[8] J. Bloch, J. Glesaaen, J. J. M. Verbaarschot, and S. Zafeiropoulos, J. High Energy Phys. 03 (2018) 015.
[9] I.-O. Stamatescu (unpublished).

[10] J. Berges and D. Sexty, Nucl. Phys. B799, 306 (2008).

[11] J. Berges, S. Borsanyi, D. Sexty, and I.-O. Stamatescu, Phys. Rev. D 75, 045007 (2007).

[12] C. Pehlevan and G. Guralnik, Nucl. Phys. B811, 519 (2009).

[13] K. Nagata, J. Nishimura, and S. Shimasaki, Phys. Rev. D 94, 114515 (2016).

[14] A. C. Loheac and J.E. Drut, Phys. Rev. D 95, 094502 (2017).

[15] F. Attanasio and B. Jäger, Eur. Phys. J. C 79, 16 (2019).

[16] E. B. Davies, Linear Operators and Their Spectra (Cambridge University Press, Cambridge, UK, 2007). 